\title{
Left heart hypoplasia operated using double pulmonary arterial banding with double arterial duct stenting: A case report
}

\author{
CRISTINA DRAGOMIR ${ }^{1}$, ANIKO-MARIA MANEA ${ }^{1,2}$, VIRGIL RADU ENATESCU $^{3}$, \\ ALINA ADRIANA MARA LACATUSU ${ }^{4}$, ADRIAN LACATUSU ${ }^{4,5}$, \\ OSAKWE IFEANYI HENRY ${ }^{6}$, MARIOARA BOIA ${ }^{1,2}$ and CONSTANTIN ILIE ${ }^{1,7}$ \\ ${ }^{1}$ Obstetrics-Gynaecology (XII) Department, Discipline of Neonatology and Childcare, 'Victor Babes' University of \\ Medicine and Pharmacy, 300041 Timisoara; ${ }^{2}$ Neonatology-Prematures Department, 'Louis Tुurcanu' Emergency \\ Clinical Hospital for Children, 300011 Timisoara; ${ }^{3}$ Neuroscience (VIII) Department, Discipline of Psychiatry, \\ 'Victor Babes' University of Medicine and Pharmacy, 300041 Timisoara; ' ${ }^{4}$ Bega' Pediatric II Clinic, \\ 'Pius Brinzeu' County Emergency Clinical Hospital, 300226 Timisoara; ${ }^{5}$ Pediatrics (XI) Department, \\ Pediatrics II Discipline, 'Victor Babes' University of Medicine and Pharmacy, 300041 Timisoara; \\ ${ }^{6}$ Pediatric Surgery and Orthopedics Department, Arad County Emergency Clinical Hospital, 310158 Arad; \\ ${ }^{7}$ Neonatology Department, 'Pius Brinzeu' County Emergency Clinical Hospital, 300226 Timisoara, Romania
}

Received July 10, 2020; Accepted August 10, 2020

DOI: $10.3892 / \mathrm{etm} .2020 .9323$

\begin{abstract}
Hypoplastic left heart syndrome is a heterogeneous group of congenital cardiac malformations which associates hypoplastic/aplastic left ventricle, mitral and aortic valve, hypoplastic/atresia and severe aortic artery coarctation, and represents a medical-surgical emergency. We present a case of a newborn hospitalised in three clinics (two clinics from Timisoara and one from Vienna), and operated for hypoplastic left heart syndrome, without aortic coarctation, using a mixed technique cardiovascular repair surgery. The initial therapeutic conduct included maintaining the permeability of the arterial canal with prostaglandin E1. At the Vienna General Hospital, at the age of 17 days, bilateral banding of the pulmonary artery was performed and, at the age of 20 days, during the cardiac catheterisation, the Rashkind procedure (balloon atrial septostomy) was performed, with two stents being implanted in the arterial canal. Postoperative complications were postcardiotomy syndrome, pneumonia with Enterococcus faecalis and Stenotrophomonas maltophilia, sepsis with methicillin-resistant Staphylococcus aureus, coagulopathy, mixed anaemia, and metabolic acidosis. The patient died 1 month after the intervention due to cardiorespiratory arrest, bilateral congestive heart failure, left heart hypoplasia with shunt through the arterial
\end{abstract}

Correspondence to: Dr Cristina Dragomir or Dr Aniko-Maria Manea, Obstetrics-Gynaecology (XII) Department, Discipline of Neonatology and Childcare, 'Victor Babes' University of Medicine and Pharmacy, 2 Eftimie Murgu Square, 300041 Timisoara, Romania E-mail: dr.cristinadragomir@yahoo.com

E-mail: aniko180798@yahoo.com

Key words: catheterisation, left heart hypoplasia, persistent arterial duct, postcardiotomy syndrome, pulmonary arteries banding canal and pulmonary artery banding, multiorgan failure, and severe secondary haemorrhagic disease. In conclusion, the initial cardiac surgical reconstruction consisted of a mixed technique, and anticoagulant medical treatment with heparin, antibiotics (bacterial endocarditis prophylaxis to be performed throughout life); postintervention hypoxic and infectious complications resulted in multiorgan failure and death.

\section{Introduction}

Hypoplastic left heart syndrome (HLHS), initially described by Maurice Lev (1952), is a heterogeneous group of congenital cardiac malformations (CCMs) that associates: The hypoplastic/aplastic left ventricle (LV), the hypoplastic/atresia mitral and aortic valve (M/AoV), and the aortic artery coarctation (AoAC) in a severe form $(1,2)$. The incidence of HLHS is $0.16-0.30 \%$ of live births in the USA, and $0.22-0.37 \%$ of live births in the UK $(3,4)$. There are no studies for Romania that record the exact incidence of HLHS. It represents 1.2-1.5\% (up to $3 \%$ ) of CCMs, and 7-9\% of congenital heart diseases diagnosed in the first year of life; before the surgical intervention, it caused $25 \%$ of deaths because of neonatal heart disease (5-7). Boys represent 55-70\% of cases. The exact cause of the disease is unknown, but it has been postulated that it has a multifactorial transmission. There are family cases transmitted recessively and autosomally; in 5-15\% of cases, HLHS is included in genetic syndromes, such as Turner, Noonan, Smith-Lemli-Opitz or Holt-Oram, Edwards (trisomy 18), Patau (trisomy 13), Jacobson (chromosome deletion 11q), Rubinstein-Taybi and partial trisomy $9(1,8,9)$.

\section{Case report}

We present the case of a child with HLHS born at 'Bega Maternity' in Timişoara, operated using a mixed technique 

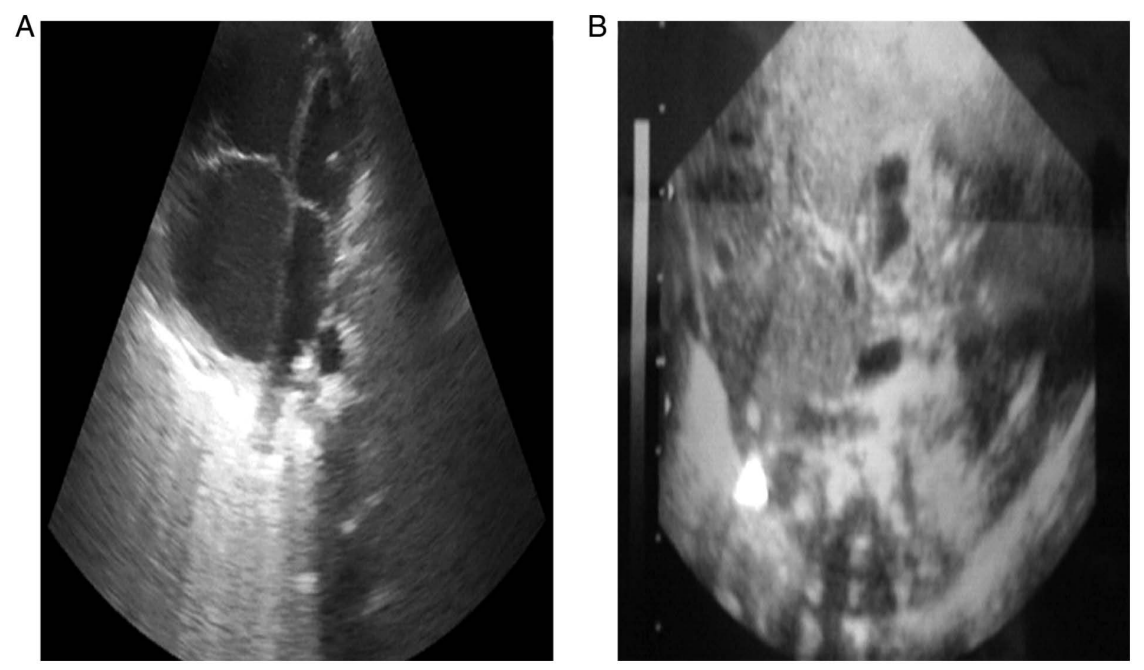

Figure 1. Echography: (A) Echocardiography in the first day of life: Left heart hypoplasia. (B) Abdominal ultrasound: Gallbladder with minimal biliary sludge, ascites (perihepatic, in the Morison space, minimal peri splenic, in the bilateral parietal-colic nodes), normal cord diameter in the hilum.

cardiovascular repair surgery at the Vienna General Hospital, and treated following the intervention at the 'Louis Tुurcanu' Emergency Clinical Hospital for Children-Neonatology and Prematurity Unit from Timişoara. The study was conducted in line with the CARE criteria, following the CARE guidelines: Consensus-based clinical case report guideline development. Ethics approval was obtained from the Research Ethics Committee of the 'Louis Thurcanu' Emergency Clinical Hospital for Children in Timisoara (no. 3697/05.03.2020). Consent to participate was also obtained.

Patient's data were retrieved from the child's observation charts. The patient aged 1 month and 1 week, having a 35-year old mother, gravida 2 para 2 (G2 P2), dispensary pregnancy (without an antenatal diagnosis of CCMs), was born by caesarean section (for indication: Risk of uterine rupture on a scarred uterus, previous caesarean delivery) at the gestational age of 39 weeks, having a birth weight of $3750 \mathrm{~g}$, a length of $51 \mathrm{~cm}$, an APGAR score of 8/1 min (cyanosis), 9/5 min. The newborn had a good early neonatal adaptation. After birth, the umbilical cord was cut, and the infant was taken to the neonatal intensive care unit, in a servo control-heated incubator, fed with free oxygen with a 2-4 1/min flow. In the first $24 \mathrm{~h}$ of life, the newborn was in good clinical condition. After $24 \mathrm{~h}$ of life, the disease manifested itself through a severe general condition, hypothermia (skin temperature of the neonate below the normal values of $36.0-36.5^{\circ} \mathrm{C}-96.8-97.7^{\circ} \mathrm{F}$, and below the normal rectal temperature of $36.5-37.5^{\circ} \mathrm{C}-97.7-99.5^{\circ} \mathrm{F}$ ), loss of appetite, skin mildly jaundiced on a pale-earthy background, declining oedema, respiratory functional syndrome, mixed dyspnoea, precordial cardiac breath grade IV/VI (auscultation), abdominal meteorism, bilious gastric residue, oligo-/anuria (urine output of $<1 \mathrm{ml} / \mathrm{kg} / \mathrm{h}$ or the absence of urinary output during the first 24 to $48 \mathrm{~h}$ of age), $3 / 3 \mathrm{~cm}$ anterior fontanelle slightly convex, lethargic, diminished archaic reflexes; bilateral or global congestive heart failure (characterised by tachypnoea, tachycardia, increased respiratory effort, rales, hepatomegaly, oedema and delayed capillary refill), as a result of a reduced right ventricle (RV) volume and pressure, and shock.
Following the anamnesis, the clinical examination and the paraclinical examinations, laboratory analysis, electrocardiogram (EKG), echocardiography, abdominal ultrasound, empty abdomen radiograph, cardiopulmonary radiograph, and thoracic computed tomography angiography, a diagnosis was established: Left heart hypoplasia (LHH), mitral valve stenosis, aortic valve stenosis, persistent arterial duct (PAD), atrial septal defect (ASD). The diagnosis of congenital heart malformation was established in the first $24 \mathrm{~h}$ of life, not antenatally (Fig. 1). The arterial duct (AD) permeability with prostaglandin E1 (PGE1) was maintained (infusible Alprostadil, $200 \mathrm{ng} / \mathrm{kg} / \mathrm{min}$, then 50 , and $15 \mathrm{ng} / \mathrm{kg} / \mathrm{min}$, respectively).

The newborn was transferred to the Vienna General Hospital. At 17 days old, bilateral pulmonary arteries (PAs) banding was performed.

At 20 days old, cardiac catheterisation was performed using the Rashkind manoeuvre (balloon atrial septostomy), and two stents were implanted in the AD. Anticoagulant therapy (heparin-dose of $28 \mathrm{IE} / \mathrm{kg} / \mathrm{h}$, infusion; acetylsalicylic acid-dose of $4 \mathrm{mg} / \mathrm{kg} / \mathrm{day}$, oral), intravenous broad-spectrum antibiotics depending on the antibiogram (ceftriaxon $100 \mathrm{mg} / \mathrm{kg} / \mathrm{day}$ and gentamicin $5 \mathrm{mg} / \mathrm{kg} / \mathrm{day}, \mathrm{IV}, 3$ days; meropenem $50 \mathrm{mg} / \mathrm{kg} / \mathrm{day}$ and amikacin $15 \mathrm{mg} / \mathrm{kg} / \mathrm{day}$, IV, 10 days; piperacillin/tazobactam 100/12.5 mg/kg/dose, sulfamethoxazole/trimethoprim $8 \mathrm{mg} / \mathrm{kg} /$ day-trimethoprim, IV infusion, caspofungin $2 \mathrm{mg} / \mathrm{kg} / \mathrm{day}$, IV infusion, 14 days; ticarcillin/clavulanate $100 \mathrm{mg} / \mathrm{kg} / \mathrm{dose}, 4$ dose $/ \mathrm{day}$, IV infusion, and ciprofloxacin $10 \mathrm{mg} / \mathrm{kg} / \mathrm{day}$, IV infusion, 10 days, and gentamicin $5 \mathrm{mg} / \mathrm{kg} / \mathrm{day}, \mathrm{IV}, 2$ days and micafungin $6 \mathrm{mg} / \mathrm{kg} / 1 \mathrm{st}$ day- $4 \mathrm{mg} / \mathrm{kg} / \mathrm{day}$, IV, 2 days), volume-expanders, blood derivatives (erythrocyte concentrate, fresh plasma, cryoprecipitate), immunoglobulin (for indications: Recurrent neonatal infections and thrombocytopenia, dose of $0.4 \mathrm{~g} / \mathrm{kg} / \mathrm{day}, 3$ days, infusion), inotropic drugs (adrenaline-for indication: Bradycardia, 0.01-0.03 mg/kg/dose, IV push, and $0.2 \mathrm{mcg} / \mathrm{kg} / \mathrm{min}$, IV infusion; atropine-for indication: Bradycardia, dose 0.01-0.03 mg/kg IV), amino acids (for indication: Hypoproteinaemia), corticosteroids, antihypertensives (enalapril $0.02 \mathrm{mg} / \mathrm{kg} / \mathrm{day}$ and clonidine $0.02 \mathrm{mg} / \mathrm{kg} / \mathrm{day}$, oral), 

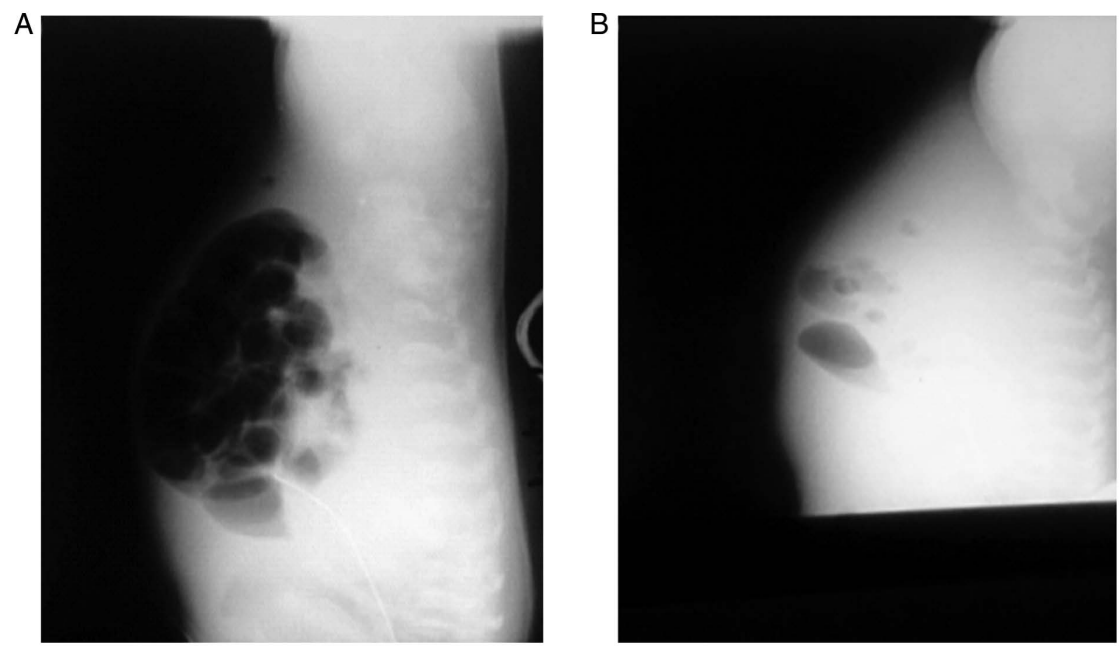

Figure 2. Empty abdomen X-ray: (A) Airway, without hydro-aerial levels, without pneumoperitoneum, ventilation absent in the small basin. (B) Relaxed abdomen with poorly visible safety lysate, reduced ventilation, without intestinal hydroelectric levels, ascites.
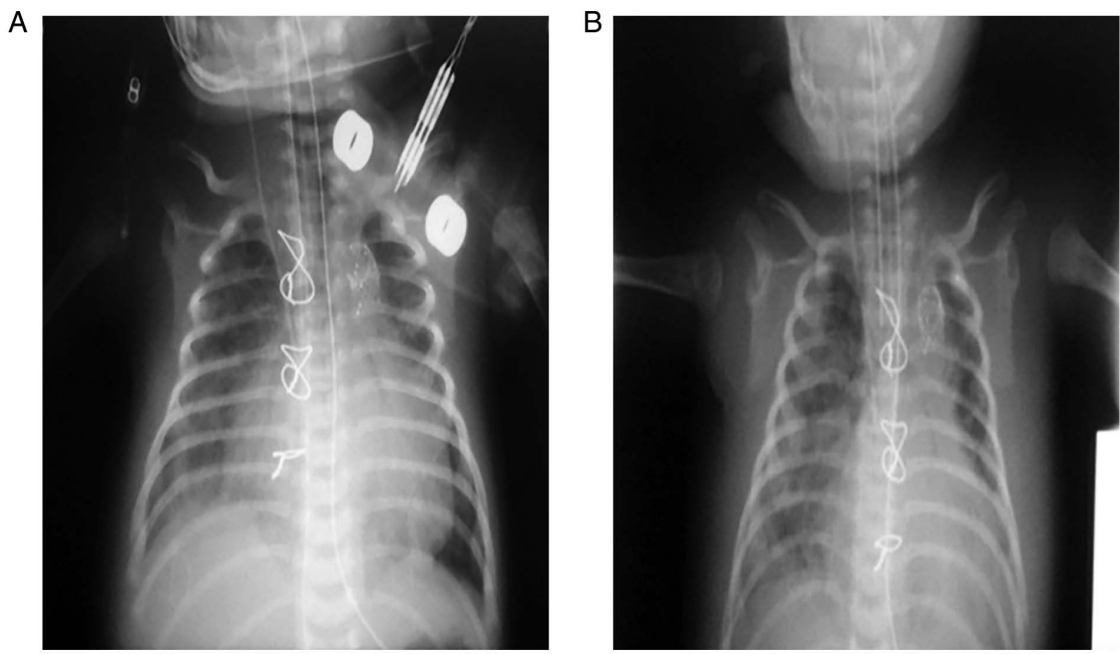

Figure 3. Cardio-pulmonary radiography: (A) Postoperative status for hypoplastic left heart syndrome, cardiomegaly, post-sternotomy circles, pericardial drainage tube. (B) Post-intervention status for hypoplastic left heart hypoplasia; acute pneumonia, cardiomegaly.

diuretics, proton pump inhibitors, vitamin $\mathrm{K}$ and mannitol, were used.

At 7 days following the intervention, the child was hemodynamically stable. The echocardiography showed LHH, a good systolic function of systemic RV, grade I-II tricuspid regurgitation, grade I pulmonary valve regurgitation, bilateral PAs banding with saw-tooth systolic-diastolic doppler flow, patent double ductal stent, right-left shunt $2.1 \mathrm{~m} / \mathrm{s}$, very good flow in descending AoA, retrograde flow in AoA $1.8 \mathrm{~m} / \mathrm{s}$, antegrade flow via very thin AoV, open, pericardial fluid $4-5 \mathrm{~mm}$, patent foramen ovale (PFO) type ASD (left-right shunt $0.8 \mathrm{~m} / \mathrm{s}$ ), and pericarditis with pericardial drainage.

At 10 days following the intervention, the clinical condition worsened, the child displaying increased cardiomegaly with pulmonary stasis. The abdomen was relaxed with poorly visible safety lysate and ascites (Fig. 2). The following were performed: Pericardial drainage (12 days), left pleural drainage (6 days), right pleural drainage (5 days). The postprocedural echocardiography was favourable: Dilated RA, ASD (following dilatation with a balloon), normal LA, RV with conserved contractility and ejection fraction (EF) $42 \%$. LV is rudimentary. The AoA diameter at the ring was $4 \mathrm{~mm}$ and was retrogradely loaded. The AD stent was permeable. After the surgery at the Vienna General Hospital, 1-month old baby was transferred to the Neonatology Clinic in Timisoara.

At 14 days following the intervention: Pericardial drainage was suppressed, but the clinical condition was severe, with earthy teguments and generalised oedema. Echocardiography showed good RV function, tricuspid regurgitation $(\operatorname{Vmax}=5 \mathrm{~m} / \mathrm{s}$, band on efficient PAs branches, stents on $A D(V \max =2 \mathrm{~m} / \mathrm{s})$, laminar flow AoA hypoplasia. Clinically, functional respiratory syndrome, melena, and severe metabolic acidosis appeared (Table I). The newborn required orotracheal reintubation and mechanical ventilation.

At 21 days following the intervention: Massive oedema with thoracic infiltration. During the chest auscultation, bilateral sub-repeating rales were detected. Pulmonary radiography showed acute pneumonia (Fig. 3). Mild (beneficial) respiratory alkalosis was maintained. Hypertension was more commonly caused by increased pulmonary blood flow (at the expense of 


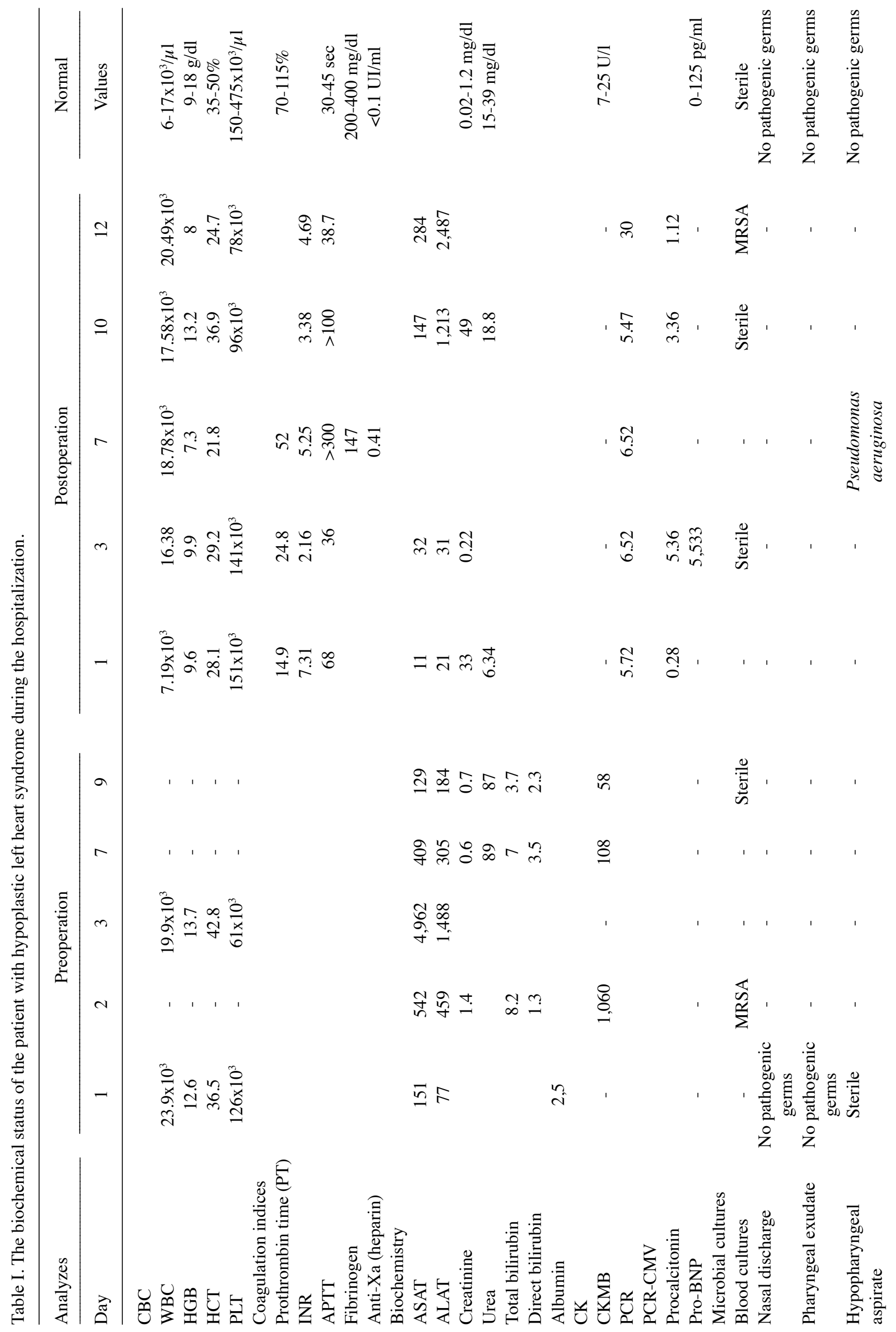




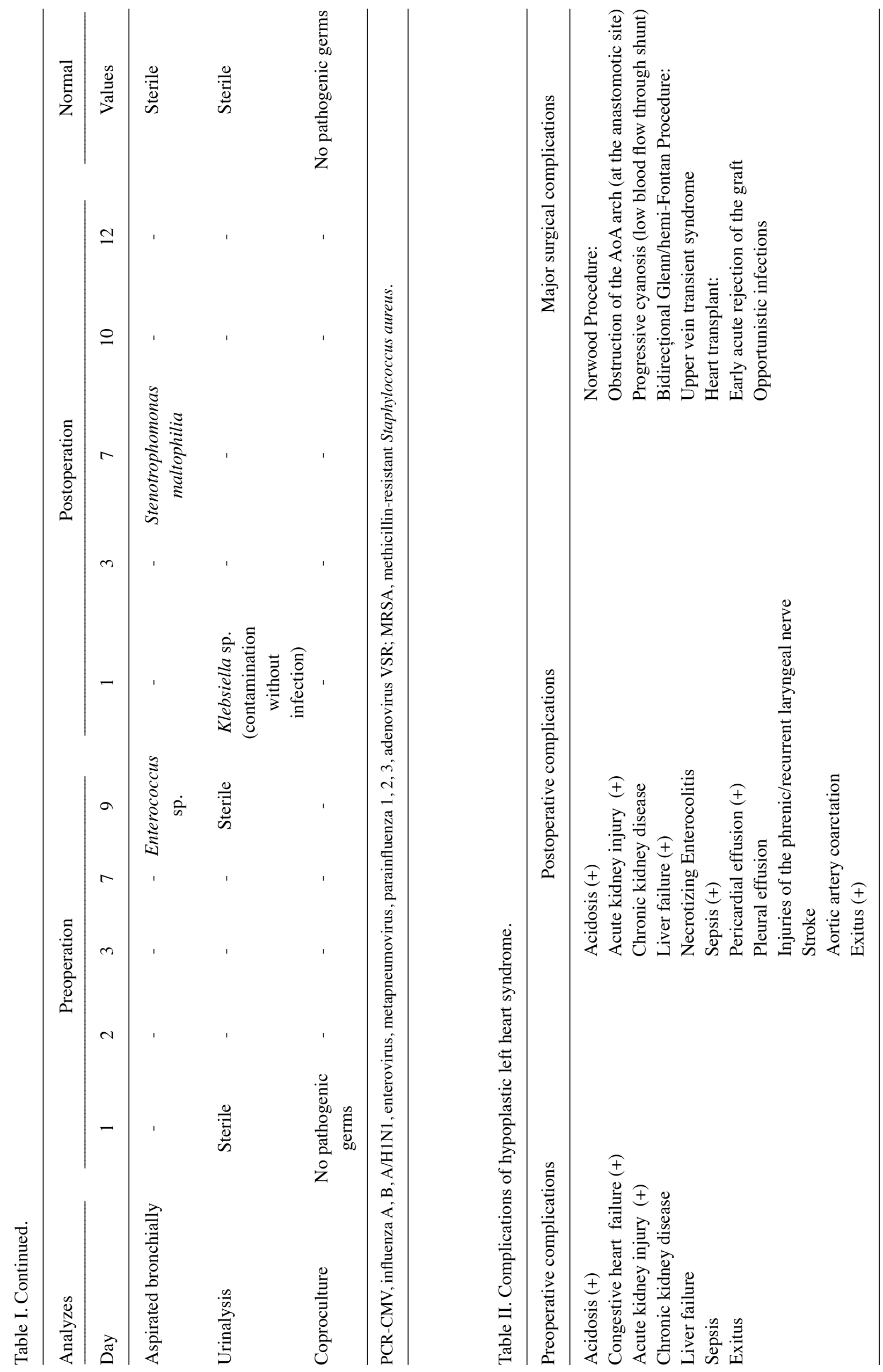


systemic flow) than intrinsic myocardial dysfunction. After extubation, the patient was reintubated orotracheally and mechanically ventilated. Following the surgery, the complications were postcardiotomy syndrome, pneumonia with Enterococcus faecalis and Stenotrophomonas maltophilia, sepsis with methicillin-resistant Staphylococcus aureus (at 21 days post-surgery in the neonatal clinic in Timisoara), coagulopathy, mixed anaemia, and metabolic acidosis (Table II). The patient died one month after the intervention due to cardiorespiratory arrest, bilateral CHF, HLHS with AD shunt and PAs banding, multiorgan failure, and severe secondary haemorrhagic disease.

\section{Discussion}

Clinical and haemodynamic forms include: 1) LV and MV and AoV atresia/hypoplasia; Hypoplastic ascending and transverse AoA, frequently associated with AoAC; the flow through the coronary arteries is often retrograde from the AD into the small ascending AoA; 2) another variant of HLHS, similar to the one presented, also display narrow PAD $(8,9)$. The case studied falls into the latter form.

The newborn with HLHS may benefit from infusion with prostaglandin E1 (PGE1) if he or she has a PFO; when foramen ovale is low/absent, clinical status is critical, and will not improve after PGE1. The patient in the study was infused with PGE1. Acute vascular collapse secondary to AD closure induces shock (8-10). The mandatory surgical treatment to be performed is reconstruction through a series of three surgical interventions: Stage I, the Norwood procedure (at 2 weeks of age), stage II, Hemi-Fontan or the two-way Glenn procedure (at the age of 6-9 months), and stage III, modified Fontan/Fontan procedure (at the age of 18-24 months); orthotopic heart transplant, initially performed by L. Bailey, has similar results (11-13). The mixed technique performed at Vienna General Hospital was performed safely.

The overall survival after stage I, mentioned in the literature, is $75-93 \%$ when the RV's function is normal preoperatively, and $47 \%$, when the RV is dysfunctional $(5,6,12)$. Negative prognostic elements are high Aristotle comprehensive complexity score (ACCS) $>20$ points (a useful tool for the analysis of the outcome after congenital heart surgery, with values between 1.5 and 25 points; it included two categories of complexity factors: Procedure dependent factors and procedure independent factors), low oxygen saturation in the first $48 \mathrm{~h}$ after stage I (14). The severe metabolic acidosis, progressive oedema and post-surgical infections caused an unfavorable prognosis (in the case studied). Survival after Glenn/Hemi-Fontan and Fontan bidirectional operations is $90-95 \%$ (15). The survival rate at 5 years after the reconstruction in stages is $70,20 \%$ of infants on cardiac transplant lists die preoperatively, and the survival rate at 5 years after the transplant is $80 \%$; the Society of Congenital Heart Disease Surgery shows that the survival rate of patients operated by the RV-PAs shunt Norwood technique is the highest, and the HYBRID technique has better results only in low birthweight infants (16).

In conclusion: i) in the studied case, the HLHS is a CCM that associates MV stenosis, AoV stenosis, PAD, ASD, and represents a medical-surgical emergency; ii) the AD patent with PGE1 is maintained in a continuous infusion, to allow pulmonary venous blood to pass through the shunt from the left atrium to the right atrium; iii) the initial treatment consists of a mixed technique: Pas bilateral banding (at 17 days old), followed by cardiac catheterisation using the Rashkind manoeuvre (balloon atrial septostomy), and implanting two stents in the AD (at 20 days old); iv) medical treatment includes anticoagulant therapy with heparin, antibiotics (bacterial endocarditis prophylaxis to be performed throughout life), volume-expansion, blood products, inotropic agents, corticosteroid therapy, diuretics, and mechanical ventilation; v) post-surgery complications (acute pneumonia with Enterococcus faecalis and Stenotrophomonas maltophilia, severe metabolic disorders, toxic-septic status, and massive pulmonary and digestive bleeding) have aggravated the patient's condition; vi) one month after the surgery, death occurs by cardio-respiratory arrest, global congestive heart failure, HLHS with shunt by AD and PAs bilateral banding, multiorgan insufficiency, and severe secondary haemorrhagic disease; vii) the prenatal diagnosis should lead to delivery in a specialized center, and improving the prognosis.

\section{Acknowledgements}

The authors would like to thank Dorel-Viorel Cretu and Sebastian Adam Puraci-technical for their support.

\section{Funding}

No funding was received.

\section{Availability of data and materials}

All data generated or analyzed during this study are included in this published article.

\section{Authors' contributions}

CD, AMM, VRE, AAML, AL and OIH were involved in the conception and design of the study, acquired, analysed and interpreted the data, and drafted the manuscript. MB was involved in the conception of the study, analysed and interpreted the data, and reviewed the manuscript. CI was involved in the conception of the study, coordinated the study, reviewed the manuscript and gave final approval of the version to be published. All authors read and approved the final manuscript.

\section{Ethics approval and consent to participate}

The study was conducted in line with the CARE criteria, following the CARE guidelines: Consensus-based clinical case report guideline development. Ethics approval was obtained from the Research Ethics Committee of the 'Louis Tुurcanu' Emergency Clinical Hospital for Children in Timisoara, Romania (no. 3697/05.03.2020). Consent to participate was also obtained.

\section{Patient consent for publication}

Not applicable. 


\section{Competing interests}

The authors declare that they have no competing interests.

\section{References}

1. Si MS, Hirsch-Romano JC, Bove EL, Ohye RG, Windle ML, Mancini MC, Berge S and Odim J: Surgical treatment of pediatric hypoplastic left heart syndrome. Medscape, 2014.http://emedicine medscape.com/article/904137-overview. Accessed April 11, 2014

2. Si MS, Bove EL, Romano JC and Ohye RG: How I teach the Norwood procedure. Ann Thorac Surg 101: 2045-2048, 2016.

3. Cloherty J, Eichenwad E and Stark A: Manual of neonatal care. 7th edition. Lippincott Williams \& Wilkins, Philadelphia, PA, 2017.

4. Gardner S, Carter B, Enzman-Hines M and Hernandez J: Merenstein and Gardner's Handbook of neonatal intensive care. 8th edition. Elsevier Inc., St. Louis, MO, 2016.

5. Sakurai T, Rogers V, Stickley J, Khan N, Jones TJ, Barron DJ and Brawn WJ: Single-center experience of arch reconstruction in the setting of Norwood operation. Ann Thorac Surg 94: 1534-1539, 2012.

6. Anderson R, Pozzi M and Hutchinson S: Hypoplastic left heart syndrome. Springer-Verlag, London, 2005.

7. Alabdulgader AA: Survival analysis: Outcome of prenatal versus postnatal diagnosis of hypoplastic left heart syndrome. J Invasive Cardiol 1: 8-12, 2018.
8. Roeleveld PP, Axelrod DM, Klugman D, Jones MB, Chanani NK, Rossano JW and Costello JM: Hypoplastic left heart syndrome: From fetus to Fontan. Cardiol Young 28: 1275-1288, 2018.

9. Beer M and Berkow R: The Merck manual of diagnosis and therapy, 18th edition. Merck, 2006.

10. Connor JA and Thiagarajan R: Hypoplastic left heart syndrome. Orphanet J Rare Dis 2: 23, 2007.

11. Freedom R, Benson L and Smallhorn J: Neonatal heart disease. 1st edition. Springer-Verlag, London, 1991.

12. Johnson S: Hypoplastic left heart syndrome. Healthline Media, San Francisco, CA, 2016. https://www.healthline.com/health/ hypoplastic-left-heart-syndrome. Updated March 30, 2017.

13. Riddick-Grisham S: Pediatric life care planning and case management. Vol 3. 2nd edition. CRC Press LLC, Boca Raton, FL, 2011.

14. Erek E, Yylmaz B, Kaya M, Onan YS, Pen O, Oz K, Koçyigit ÖI, Güzeltaş A and Ödemiş E: Analysis of results according to the Aristotle scoring system in congenital heart surgery. Turkish J Thoracic Cardiovascular Surg 22: 509-516, 2014.

15. Kliegman R, Stanton B, Geme J and Schor N: Nelson Textbook of pediatrics. Vol 2. 20th edition. Elsevier Inc., 2016.

16. Wilder T, Hickey E, Ziemer G, Jacobs M, Gruber P, Blackstone E, McCrindle B, Williams W, DeCampli W, Caldarone AC and Pizarro C: Hybrid Alternatives to Norwood Stage-1 are not a lower risk alternative: Norwood-RVPA offers better outcome in comparable neonates. Circulation 130: 19493, 2018. 